\title{
Intelligent Feature Extract System for Cursive-Script Recognition
}

\author{
Khalid Saeed and Marek Tabedzki \\ Computer Science Department \\ The University of Finance and Management \\ in Bialystok, Poland \\ Faculty of Computer Science \\ Bialystok Technical University \\ Poland \\ http://aragorn.pb.bialystok.pl/ zspinfo/
}

Summary. The paper describes a newly presented hybrid method for high efficiency in script image feature extraction. The recognition rate was about $82 \%$ for very large number of scripts per class. However, it has reached even $100 \%$ in some cases with a smaller number of scripts per class. The system contains two projectionbased methods for image characteristics extraction presented by very simple feature vectors and one image descriptor. A specially worked out thinning algorithm for the recognition system has simplified the feature extracting procedure as it provides a continuous one-pixel width skeleton of the script, which is essential for the simple-projection approach.

\section{Introduction}

The problem we are focusing our work on in this paper is recognition of handwritten texts. Talking about recognition, two different issues (depending on what is supposed to be recognized) are worth mentioning: text recognition and writer identification. Text recognition stands for classifying the scripts of a given text, and in effect, converting the bitmap image into an editable text document. Writer identification, however, stands for analyzing handwritten text, and finding its author. In fact, these two issues differ only in the manner of classification. Nevertheless, they are complementary to each other. Methods used in one of them can be adapted to the other. What distinguishes them is their approach to classification, structure of database and results interpretation.

There are varieties of techniques and methods for text recognition, but there exist some common steps to almost all of them (Fig. 1). 


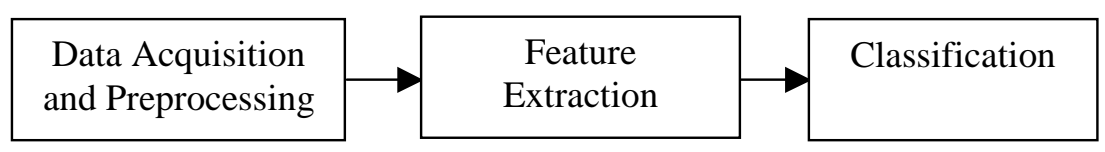

Fig. 1. Main stages of recognition process.

- Data acquiring and preprocessing - the task of producing (by digitalization) binary representation of image for recognition. Various techniques of preprocessing are being used by the authors to increase the quality of the acquired image and hence increasing the success rate of the recognition process.

- Feature extraction - extraction of those attributes of the tested image that are significant and important in the process of recognition. The main goal is the reduction of data. The other important task is to keep all the meaningful traits and features of the script. It is definitely the most significant stage in the recognition, and the success of the whole process will depend on its results. Besides, the amount of the generated data will influence the speed of classification, as well. The contribution of the authors to this task is seen in $[1,2,3]$.

- Classification - on the basis of the feature vectors, the given scripts are classified and identified as the correct ones in the given database class. The system suggested in this paper works in most cases as an extractor and a classifier at the same time.

In this work the authors have presented what they had done and modified in the field of feature extraction for image recognition. For a better and more successful rate of recognition, the authors have worked out their own ways of preprocessing through simple algorithms and methods of data acquisition and image preparation for recognition system

In the following sections we will describe each of the stages in our recognition system in details.

\section{Preprocessing}

The first stage is to acquire and preprocess the data. In effect we obtain a bitmap (2D image) for further analysis and identification. In our work we acquire images by scanning or using graphic tablets. Touch screens, cameras or other tools have also been considered but not at the same level as scanners for the purpose of this work. The acquired text was a simple sentence, handwritten by several different people with different writing styles and writing tools.

Preprocessing is particularly important in the case of scanned images, it consists of edge smoothing, noise reduction and defects removing so as to eliminate errors occurring in the original document or appearing in the stage 
of digitalization. The quality of the obtained image is of significant meaning to the whole process of recognition.

Our scanned images were binarized (converted into black and white images) by removing all levels of gray, which might appear in the original image.

Sometimes, and in most cases at least so far, it is necessary to use thinned images. Thinning process removes some redundant pixels of a shape to achieve a one-pixel-width skeleton for a character - it allows suppressing the differences between scripts written with various tools. Some methods, because of their specification, require thinned shapes, others even furnish better results for thinned characters $[4,5,6]$. One significant benefit from thinning is that it reduces the amount of information to analyze as it decreases the number of pixels belonging to the examined shape.

In our work whenever needed we use KMM algorithm [2] described in details in $[3,6]$ - writing it we paid particular attention to avoid the troubles with thick lines or rough edges of characters. Primary advantage of this method is that it gives a continuous one-pixel width skeleton for each line in a script. Both the Latin and Arabic scripts are of one, two or three lines each. Figure 2 shows some examples of thinning Arabic, Latin letters and a Chinese word.

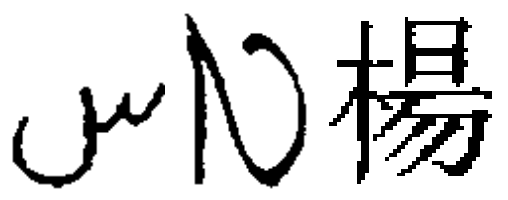

(a)

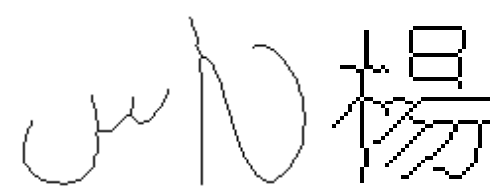

(b)

Fig. 2. Examples of thinning: Arabic letter, Latin letter and a Chinese word.

The system presented in this paper involves recognizing text through script-by-script methods analyzing each letter separately. Therefore, the segmentation is beyond the topics of this work and will be treated in future works. In fact, segmentation is substantial because in case of incorrect splitting, we are not able to identify characters properly (e.g. letter 'W' may be recognized as 'VV', or letters 'IV' as 'N'.) It can be aided by joining segmentation with algorithm of classification, and some methods rely on this feature - in case of inaccurate or disputable classification they can be retreated following the methods of segmentation or using a dictionary to classify them accordingly.

The final effect of preprocessing is obtaining a cleaned and prepared for further analysis bitmap-image displaying a single character.

\section{Feature Extraction}

The simplest recognition methods would perform classification right at this point $[6,7]$. Employing different methods of direct comparison can help in 
such a case. However, this classification employs the whole pixel matrix leading to time-consuming processing sensitive to any slight shifts or rotations of the character or any of its parts. This is in addition to the required big size of database. It must be massive enough, to assure high effectiveness of recognition, because the number of examples is always crucial - there are various writing styles, so our base should contain as many of them as possible in order not to have problems with typical variants of the given character. This essentially implies that we reduce the amount of the input data to analyze. To achieve that various statistical methods of data dimensional reduction like Principal Components Analysis or its modified versions SVD i LDA [8] can be used successfully for that purpose [9, 10]. Other developing approaches like MPEG-7 [11] can also be applied. The main advantage of those methods is generality and their mathematical efficiency. However, because of our adequate knowledge about the problem, we can use intuition estimating the importance of character features and heuristically choose which attributes to explore. Whatever the chosen method is, it will be based on building some abstract representation of the analyzed character, having less data than the input image. In the proposed approaches in this paper, the new image representation has the form of one-dimensional vector with a constant length independent of the bitmap size.

\subsection{Simple-Projection Approach}

The first of these methods is the projection approach. It consists of projecting each pixel of a character onto axes forming a grid, in which the character is inscribed in its thinned form. The thinning procedure was performed by the mentioned above KMM algorithm [2,3]. The first versions of our projection method made use of two or four projection axes (Fig. 3a and 3b). Then the approach was modified in adding more projection axes to have twelve axes (Fig. 3c and Fig. 4b). This gave good results with both Latin and other alphabets like those of cursive character (Arabic, for example) showing an optimal choice of projection number of axes sufficient to describe the thinned image [12]. The projection procedure is based on the idea that each black pixel is cast onto the nearest two of the grid-axes (one vertical and one horizontal.)

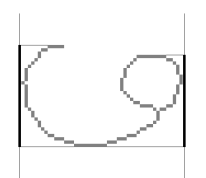

(a)

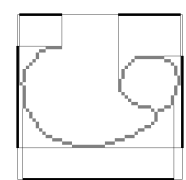

(b)

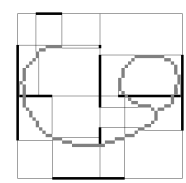

(c)

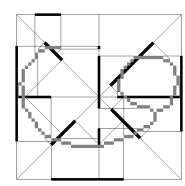

(d)

Fig. 3. Projecting an Arabic letter onto (a) 2, (b) 4, (c) 12 and (d) 13 axes. 
In his paper [13] Burr (Fig. 3d and Fig. 4a) used the projection approach through 13 axes (four of them were diagonal), and each pixel was projected onto the nearest three of them. Originally, Burr's model was used for digit recognition. Our modified projection model (Fig. 4b), however, has no diagonal axes.

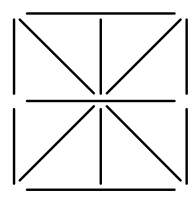

(a)

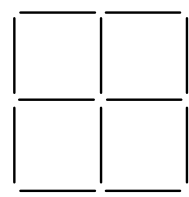

(b)

Fig. 4. The two known models of projection: Burr's (a) and the authors' (b).

Despite this, as results have proved [6], our method performs better and gives higher rate of recognition than Burr's as the results will show.

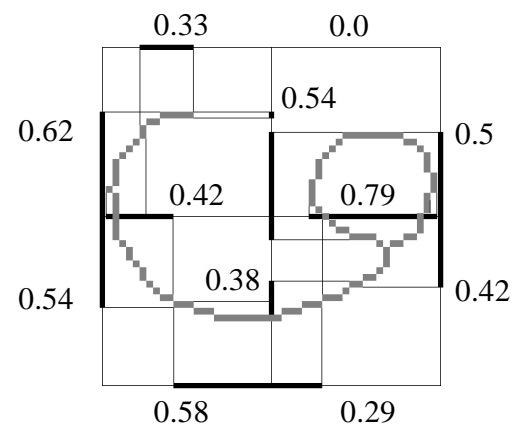

Fig. 5. The Arabic letter ق - Qqaf projected on 12 axes.

In further steps of the algorithm the percentage of coverage of each axis is calculated (Fig. 5). In this way we obtain twelve values forming the feature vector describing the given character as given in Eq. (1).

$$
F V=(0.33,0.0,0.42,0.79,0.58,0.29,0.62,0.54,0.54,0.38,0.5,0.42)
$$

\subsection{View-Based Projection Approach}

The second method presented in this paper is the view-based model. What is new in this method is no need for thinning as we do not examine the individual pixels of the image bitmap, but only the outer contour of the script. On the other hand, any interruption in the shape may affect the final result because 
the lack of continuity would cause errors in setting the contour. If these disconnections come from a particular writing style, it is not a problem as we can have a sample base that is strong enough to classify properly. Worse, if they result from an originally damaged image, or inappropriate image acquiring. In such cases these errors should be fixed at the stage of preprocessing for high precision of recognition.

This concept was modeled with the aim of applying it in recognizing whole words or signature identification in future. The approach presented in this paper is an extension to the concept based on four views of separate characters [14]. This idea arises from the fact, that we can recognize words looking at only their profiles.

The "view" was defined as a set of extreme pixels belonging to the contour of the script, for example, top view is a set of points having maximal $y$ coordinate for a given $x$ coordinate (Fig. 6).

Not all the pixels of views are examined, but only a certain fixed number of uniformly distributed characteristic points. They are marked out on the surface of each view. Then the coordinates of the points are calculated.

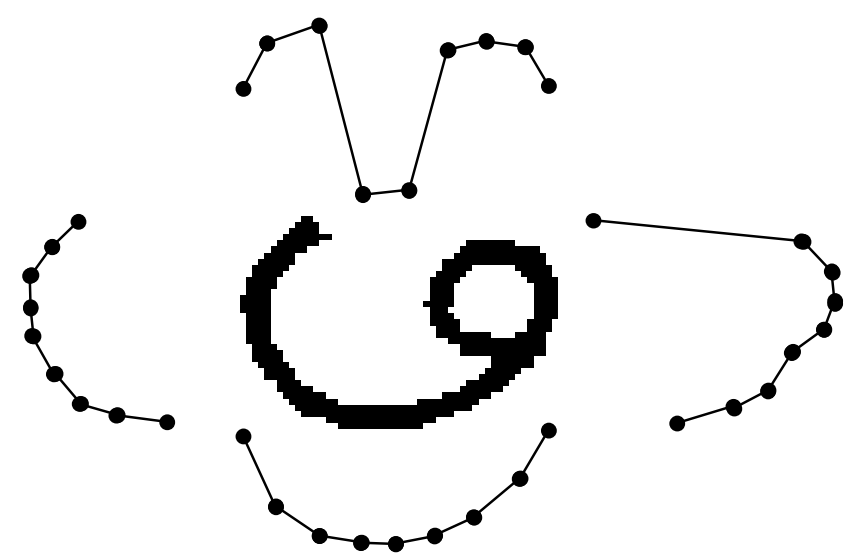

Fig. 6. Four views of the Arabic letter Qqaf "ق".

As in the case of top and down views, points are uniformly distributed along $x$ axis, we are interested only in the $y$ coordinates. The obtained values create two characteristic feature vectors describing each word or character, one for the top and the other for the bottom views. At the beginning of our experiments only a number of selected characters were examined [14]. Next experiments were performed with two further views (left and right). This was because of some of the letters like ' $E$ ' and ' $Z$ ' the system was not able to distinguish them when analyzing only the top and down views. In fact, the extended method gave very good results especially when combined with other tools of classification [15]. 


\subsection{Toeplitz-Based Descriptor}

The presented above two methods of feature extraction allow obtaining feature vectors describing a given script each. Usually it is the basis for further classification. But, we may also introduce additional stages of transformations, which will stress the characteristics of that character, for example, increase the distance between feature vectors of distinct classes, to distinguish them more easily. In our work we use the algorithm of minimal eigenvalues of Toeplitz matrices, which has proved its feasibility in many of our previous works. It gave good results for many issues related to recognition of text, voice, signature, etc. The method itself was presented and precisely described in literature $[6,7]$. In short, its application consists of processing input vector (gained from one of the above two methods,) building sequent Toeplitz matrices from its values, and calculating a series of their minimal eigenvalues. In that way we receive another vector, which describes the input data with its elements being these minimal eigenvalues of Eq. (2).

$$
F V 2=\left[\lambda_{0}, \lambda_{1}, \ldots, \lambda_{n}\right]
$$

These form a monotonically nonincreasing series (Fig. 7) carrying the charac-

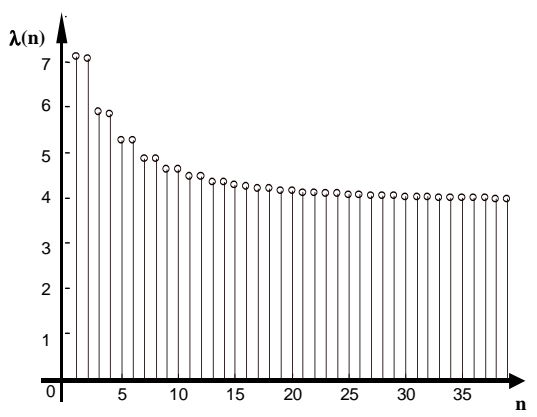

Fig. 7. The behavior of minimal eigenvalues of Toeplitz matrices.

teristics of the image they represent. The descriptor FV2 is subject to further analysis or classification. To find, calculate the modulus ci of the characteristic points coordinates to form the elements of the Toeplitz matrices $C_{i}$ :

$$
C_{i}=\left[\begin{array}{ccccc}
c_{0} & c_{1} & c_{2} & \ldots & c_{i} \\
c_{1} & c_{0} & c_{1} & \ldots & c_{i-1} \\
c_{2} & c_{1} & c_{0} & \ldots & c_{i-2} \\
\vdots & \vdots & \vdots & \ddots & \vdots \\
c_{i} & c_{i-1} & c_{i-2} & \ldots & c_{0}
\end{array}\right], \quad \text { where } i=0,1,2, \ldots, n
$$


Experiments have shown that the Toeplitz-based descriptor increased the effectiveness of recognition for some of the tested characters, while leaving others unaffected. This interesting observation led to the idea of working out a hybrid recognition system containing both of the two projection-based methods and that of minimal eigenvalues in one. As one method gives better results with some characters, and the other one with others. They can be used in parallel so that each of them makes up its own decision to give the final result as a whole (see the results in Table 1).

\section{Classification}

The final stage of the process of recognition is the classification. Now we would classify analyzed character into one of the given groups, or find which letter of the alphabet it is. The input data is the representation or description of that character, received from the given above methods. Having the aim of improving the effectiveness and efficiency of the system we have utilized artificial neural networks (ANN) for classification. It lengthens time of preparations, because of the training stage and in some cases the effectiveness may slightly decrease, in return we achieve greater performance due to saving clock time and memory space. Artificial Neural Networks give great possibility for parameterization (various types and various sizes of networks, different activation functions, variety of learning techniques, etc.). However, our main goal was to examine how the ANN would perform as a classifying tool in the combination of the presented algorithms of feature extraction in our system. We used the typical and most popular networks - three-layer feed-forward network, with sigmoid activation function shown in Eq.(4), trained with the algorithm of backpropagation. Table 1 shows the results obtained using the suggested hybrid system with these neural networks as a classifier.

$$
F(n)=\frac{1}{1+e^{-b n}}
$$

Table 1. Results comparison between the hybrid method and other approaches for some randomly selected handwritten Arabic letters.

\begin{tabular}{|c|c|c|c|c|c|c|c|c|}
\hline \multirow{2}{*}{ ClassificationMethods } & \multicolumn{8}{|c|}{ Selected Arabic Letters and their Recognition Rate } \\
\hline & ر & ص & $b$ & ف & 0 & $\hat{y}$ & g & J \\
\hline All points & 100 & 100 & 100 & 86 & 100 & 86 & 86 & 100 \\
\hline Min. Eigenvalues & 86 & 100 & 100 & 14 & 57 & 86 & 100 & 100 \\
\hline Neural Network & 73 & 100 & 96 & 84 & 100 & 76 & 96 & 100 \\
\hline Burr & 70 & 80 & 97 & 50 & 100 & 80 & 100 & 100 \\
\hline Hybrid System & 97 & 97 & 83 & 63 & 100 & 100 & 100 & 100 \\
\hline
\end{tabular}




\section{Conclusions and Recent Results}

The algorithms presented in this paper allowed attaining promising results in the case of handwritten characters identification. Applied methods of feature extraction resulted in high efficiency of recognition. Furthermore, the projection on 12 perpendicular axes has proved to be simpler in implementation than Burr's (projecting pixels onto diagonal axis is a rather more complex than onto horizontal or vertical ones). Toeplitz matrices approach combined with them in the hybrid system increased the effectiveness of recognition in the case of some characters. The use of the neural networks as classifiers has definitely improved the performance of the whole classification process.

Moreover, each of the proposed methods allows introducing further modifications and improvements, the subject of our current and future work. We are testing irregular grids in the projection approach to add an additional vector expressing the position of the projected segment on the axis, and to alter the distribution of the characteristic points in the view-based approach. The data base has been enlarged to have about 2000 samples for 16 classes. It contains all Arabic scripts written by different people not necessarily knowing Arabic alphabet. In addition, some of the letters in the base were taken from damaged handwritten Arabic texts or ones of very poor quality. Table 2 shows examples for some of the recognition results of such scripts selected for the aim of comparison, some of which were given in Table 1.

Table 2. The latest results of using the hybrid method in Arabic script recognition for selected handwritten Arabic letters (for larger data base).

\begin{tabular}{|c|c|c|c|c|c|c|c|c|}
\hline \multirow{2}{*}{ ClassificationMethods } & \multicolumn{8}{|c|}{ Selected Arabic Letters and their Recognition Rate } \\
\hline & 2 & ر & ص & b & ف & 0 & r & J \\
\hline All points & 96 & 93 & 65 & 98 & 73 & 100 & 96 & 86 \\
\hline Min. Eigenvalues & 43 & 75 & 58 & 90 & 38 & 76 & 80 & 85 \\
\hline Neural Network & 83 & 91 & 86 & 91 & 85 & 96 & 100 & 81 \\
\hline Burr & 91 & 90 & 86 & 90 & 86 & 93 & 98 & 40 \\
\hline Hybrid System & 63 & 95 & 71 & 93 & 65 & 88 & 93 & 93 \\
\hline
\end{tabular}

\section{References}

1. Saeed K., Niedzielski R., "Experiments on Thinning of Cursive-Style Alphabets," Proceedings of International Conference on Information Technologies and ITESB'99, pp. 45-49, Minsk 1999.

2. Saeed K., "Text and Image Processing: Non-Interrupted Skeletonization," Advances in Signal Processing and Computer Technologies, pp. 350-354, CSCC/IEEE, WSES Press: Crete 2001. 
3. Saeed K., Rybnik M., Tabdzki M., "Implementation and Advanced Results on the Non-interrupted Skeletonization Algorithm," CAIP'01, Lecture Notes in Computer Science, W. Skarbek (Ed.), pp. 601-609, LNCS 2124, SpringerVerlag Heidelberg 2001.

4. Chen Y.S., "The Use of Hidden Deletable Pixel Detection to Obtain BiasReduced Skeletons in Parallel Thinning," IEEE Proceedings of International Conference on Pattern Recognition - ICPR'96, Vol.II-B, pp.91-95, Vienna'96.

5. Ahmed M., Ward R., "A Rotation Invariant Rule-Based Thinning Algorithm for Character Recognition," IEEE Transactions on Pattern Analysis and Machine Intelligence, Vol.24, No.12, pp. 1672-1678, 2002.

6. Saeed K., "Image Analysis for Object Recognition," Bialystok Technical University Press, 2004

7. Saeed K., "Computer Graphics Analysis: A Method for Arbitrary Image Shape Description," MGV - International Journal on Machine Graphics and Vision, Polish Academy of Sciences, Vol.10, No.2, pp.185-194, 2001.

8. Fukunaga K., "Introduction to Statistical Pattern Recognition," Academic Press, San Diego, California, 1990.

9. Kukharev G., "Biometric Systems - Methods and Approaches of Human Personality Identification," Politechnic Press (in Russian), Petersburg 2001.

10. Skarbek W. "Tutorial on Face Recognition by Linear Discriminant Analysis," Computer Information Systems and Applications, Vol.1, pp.13-36, WSFiZ Press, Bialystok, Poland 2004.

11. Martinez J. M. (Editor), "MPEG-7 Overview," International Organization for Standardization, Pattaya, March 2003.

12. Saeed K., "A Projection Approach for Arabic Handwritten Characters Recognition," Quo Vadis Computational Intelligence? New Trends and Applications in Comp. Intell., pp. 106-111, Physica-Verlag: Berlin 2000.

13. Burr D.J., "Experiments on Neural Net Recognition of Spoken and Written Text," IEEE Tr., Acoustic, Speech and Signal Processing, Vol.36, July 1988.

14. Saeed K., Tabdzki M., Adamski M., "A New Approach for Object-Feature Extract and Recognition," 9th International Conference on Advanced Computer Systems - ACS'02, pp. 389-397, 23-25 October, Poland 2002.

15. Saeed K., Tabdzki M., "Cursive-Character Script Recognition using Toeplitz Model and Neural Networks," ICAISC'04, Lecture Notes in Computer Science, L. Rutkowski, J. Siekmann, R. Tadeusiewicz, L. Zadeh (Editors), SpringerVerlag Heidelberg, Vol. 3070, pp. 658-663, Berlin 2004. 